\title{
Gravidität bei vorbestehendem Diabetes (Update 2019)
}

\author{
Alexandra Kautzky-Willer · Jürgen Harreiter · Yvonne Winhofer-Stöckl · Raimund Weitgasser · Monika Lechleitner
}

Online publiziert: 12. April 2019

(c) Der/die Autor(en) 2019

Zusammenfassung Bereits vor 30 Jahren forderte die St. Vincent Deklaration, dass Schwangere mit vorbestehendem Diabetes mellitus vergleichbare Schwangerschaftsergebnisse wie gesunde Frauen erreichen sollen. Dennoch bestehen bei Frauen mit konzeptionell manifestem Diabetes nach wie vor höhere Komplikationsraten und eine höhere perinatale Morbidität und Mortalität. Eine fehlende oder zumindest unzureichende Schwangerschaftsplanung und präkonzeptionelle Betreuung mit Optimierung der Stoffwechsellage vor Konzeption sind dafür verantwortlich. Alle Frauen mit Diabetes sollen im Selbstmanagement der Insulintherapie mit Anpassungen der Insulindosis geschult sein und eine stabile Stoffwechsellage vor Schwangerschaftsbeginn aufweisen. Eine Schilddrüsendysfunktion, Hypertonie oder diabetische Komplikationen vor der Konzeption sollten ausgeschlossen bzw. adäquat behandelt sein, um das Risiko einer möglichen Progression der Komplikationen und Begleiterkrankungen sowie insgesamt mütterliche und fetale Risiken $\mathrm{zu}$ minimieren. Ein Ziel der mütter-

Univ.-Prof. Dr. A. Kautzky-Willer $(\bowtie) \cdot J$. Harreiter $\cdot$

Y. Winhofer-Stöckl

Gender Medicine Unit, Klinische Abteilung für

Endokrinologie und Stoffwechsel, Universitätsklinik für Innere Medizin III, Medizinische Universität Wien,

Währinger Gürtel 18-20, 1090 Wien, Österreich

alexandra.kautzky-willer@meduniwien.ac.at

R. Weitgasser

Abteilung für Innere Medizin, Privatklinik

Wehrle-Diakonissen, Salzburg, Österreich

Universitätsklinik für Innere Medizin I, LKH Salzburg -

Universitätsklinikum der Paracelsus Medizinischen

Privatuniversität, Salzburg, Österreich

M. Lechleitner

Interne Abteilung, Landeskrankenhaus Hochzirl - Natters, Hochzirl, Österreich lichen Stoffwechselkontrolle ist das Erreichen von Normoglykämie und normalen $\mathrm{HbA}_{1 \mathrm{c}}$-Werten, falls dies ohne Risiko für Hypoglykämien möglich ist, da eine schlechte Blutzuckereinstellung mit diabetischer Embryopathie und diabetischer Fetopathie assoziiert ist. Das Hypoglykämierisiko ist speziell bei Typ 1 Diabetes mellitus in der Frühschwangerschaft deutlich erhöht, nimmt aber mit den hormonellen Veränderungen und der Zunahme der Insulinresistenz im Schwangerschaftsverlauf deutlich ab. Eine weltweit steigende Adipositasprävalenz führt zusätzlich zu einem Anstieg von Müttern mit Typ 2 Diabetes. Dieser Trend ist auch bei Frauen mit Typ 1 Diabetes zu beobachten und aggraviert die Metabolik und die perinatalen Ergebnisse. Eine funktionelle, intensivierte Insulintherapie mit multiplen täglichen Insulininjektionen oder eine Insulinpumpentherapie tragen zum Erreichen einer guten mütterlichen Stoffwechselkontrolle während der Schwangerschaft bei. Orale Antidiabetika (Metformin) können vor allem bei Typ 2 Diabetes und Adipositas helfen die Insulinsensitivität zu verbessern, sollten jedoch aufgrund der Plazentagängigkeit und ungewissen Langzeitergebnissen bei den Nachkommen mit Bedacht verordnet werden.

Schlüsselwörter Präkonzeptionell bestehender Diabetes - Typ 1 Diabetes mellitus - Typ 2 Diabetes mellitus - Adipositas . Schwangerschaft . Schwangerschaftsplanung - Diabetische Embryopathie - Diabetische Komplikationen · Perinatale Morbidität

\section{Clinical practice recommendations for diabetes in pregnancy (Update 2019)}

Summary In 1989 the St. Vincent Declaration aimed to achieve comparable pregnancy outcomes in diabetic and non-diabetic women. However, currently women with pre-gestational diabetes still feature 
a higher risk of perinatal morbidity and even increased mortality. This fact is mostly ascribed to a persistently low rate of pregnancy planning and pre-pregnancy care with optimization of metabolic control prior to conception. All women should be experienced in the management of their therapy and on stable glycemic control prior to the conception. In addition, thyroid dysfunction, hypertension as well as the presence of diabetic complications should be excluded before pregnancy or treated adequately in order to decrease the risk for a progression of complications during pregnancy as well as maternal and fetal morbidity. Especially in women with type 1 diabetes mellitus in early pregnancy the risk of hypoglycemia is highest and decreases with the progression of pregnancy due to hormonal changes causing steady increase of insulin resistance. In addition, obesity increases worldwide and contributes to increasing numbers of women at childbearing age with type 2 diabetes mellitus and further deterioration of pregnancy outcomes in diabetic women. Maternal glycemic control should aim to achieve normoglycemia and normal $\mathrm{HbA}_{1 \mathrm{c}}$ levels, possibly without hypoglycemia, but is associated with the development of diabetic embryopathy and fetopathy if dysglycemia occurs. Intensified insulin therapy with multiple daily insulin injections and pump treatment are effective in reaching good metabolic control during pregnancy. Oral glucose lowering drugs (Metformin) may be considered in obese women with type 2 diabetes mellitus to increase insulin sensitivity but should be also prescribed cautiously due to crossing the placenta and lack of long-time follow up data of the offspring.

Keywords Pre-gestational diabetes - Type 1 diabetes mellitus · Type 2 diabetes mellitus · Obesity · Pregnancy · Pre-pregnancy care - Diabetic embryopathy · Diabetic complications · Perinatal morbidity

\section{Grundsatz-Statement}

In der St. Vincent Deklaration wurde 1989 als Ziel festgelegt, dass Frauen mit Diabetes in Zukunft vergleichbare Schwangerschaftsergebnisse haben sollen wie stoffwechselgesunde Frauen. Dennoch weisen Frauen mit Diabetes nach wie vor mehr mütterliche und kindliche Komplikationen und eine höhere perinatale Mortalität auf. Dies liegt vor allem an der immer noch unzureichenden Schwangerschaftsvorbereitung und Blutzuckeroptimierung zu Beginn der Gravidität. Auch während der Gravidität einschließlich der Geburt muss eine möglichst normoglykämische Stoffwechsellage gewährleistet sein. Nach Möglichkeit sollten Frauen mit Diabetes von einem in der Behandlung schwangerer Frauen mit Diabetes erfahrenen, interdisziplinären Team an einem spezialisierten Zentrum betreut werden. Die Entbindung sollte an einer Abteilung mit neonataler Intensivstation erfolgen.

\section{Schwangerschaftsplanung - perikonzeptionelle Betreuung}

Eine Schwangerschaft bei Frauen mit manifestem Diabetes betrifft immer noch hauptsächlich Frauen mit Typ 1 Diabetes mellitus (T1DM). Typ 2 Diabetes mellitus (T2DM) in der Schwangerschaft tritt aber aufgrund steigender Adipositasprävalenz im gebärfähigen Alter immer häufiger auf. Rezente nationale Erhebungen zeigen eine kontinuierliche Zunahme des T2DM, der zusätzlich zur Hyperglykämie auch durch die adipositasbedingten Risiken und oft auch durch ein höheres mütterliches Alter kompliziert wird [1, 2]. Daten der Universitätsklinik in Wien haben einen Anstieg an stark übergewichtigen graviden Frauen mit T2DM bestätigt [3], wobei selbst bei den Schwangeren mit T1DM im letzten Jahrzehnt ein signifikanter Anstieg im BMI festgestellt wurde. Sowohl bei T1DM als auch T2DM waren neben der Stoffwechselkontrolle und Diabetesdauer zu Schwangerschaftsbeginn, der mütterliche BMI und ein höherer Blutdruck mit schlechteren Schwangerschaftsergebnissen verbunden [4]. Migrantinnen sowie Frauen aus niedrigen sozialen Schichten machten einen beträchtlichen Anteil der Frauen mit T2DM aus, insbesondere bei jener Gruppe, die vor der Gravidität unzureichend behandelt und auf die Schwangerschaft vorbereitet war oder bei der ein vorbestehender Diabetes überhaupt erst in der Schwangerschaft neu entdeckt wurde.

Frauen mit Diabetes müssen - unabhängig von der Diabetesform - eine Schwangerschaft planen, um optimale Voraussetzungen für die kindliche Entwicklung, aber auch die eigene Gesundheit zu gewährleisten und das Risiko für perinatale Komplikationen zu reduzieren $[1,2]$. Frauen mit Diabetes sollten bezüglich der Verhütungsmethode dieselben Optionen wie gesunden Frauen zur Verfügung stehen, da das Risiko einer ungeplanten Schwangerschaft das Risiko der Kontrazeption übertrifft [5]. In einer großen amerikanischen Studie mit Frauen mit T1DM und T2DM konnte die Sicherheit von hormonellen Verhütungsmethoden und niedriges Risiko für thromboembolische Ereignisse (1 thromboembolischer Event pro 100 Patientinnenjahre) gezeigt werden, wobei das niedrigste Risiko bei Verwendung von intrauterinen und implantierbaren subdermalen kontrazeptiven Methoden beobachtet wurde [6]. Das Hauptproblem bei vorbestehendem Diabetes ist die Entstehung einer diabetischen Embryopathie [1, 2]. Aus diesen Gründen werden eine prinzipielle präkonzeptionelle Beratung und engmaschige Betreuung aller Frauen mit Diabetes und Kinderwunsch oder Schwangerschaft empfohlen. Um Missbildungen und Aborte $\mathrm{zu}$ vermeiden, soll der Glukosestoffwechsel mit einem $\mathrm{HbA}_{1 \mathrm{c}}<6,5 \%$, zumindest aber unter $7 \%$ bereits bei Kinderwunsch optimiert sein [5, 7]. Generell sollte versucht werden, die Patientin so gut als möglich an normoglyämische Werte zu nähern, sofern dies ohne Hypoglykämien möglich ist. Bei einem $\mathrm{HbA}_{\mathrm{lc}}$ über $8 \%$ 
Tab. 1 Risiko für kongenitale Fehlbildung und perinataler Mortalität nach $\mathrm{HbA}_{1 \mathrm{c}}$ (nach [7])

\begin{tabular}{|c|c|c|c|c|}
\hline $\mathrm{HbA}_{1 \mathrm{c}}[\%]^{*}$ & $\begin{array}{l}\text { Kongenitale Fehlbildungen } \\
(\%)\end{array}$ & $\mathrm{RR}(95 \% \mathrm{KI})^{\star \star}$ & Perinatale Mortalität (\%) & $\operatorname{RR}(95 \% \mathrm{KI})^{\star \star}$ \\
\hline$<6,9$ & 3,9 & $1,4(0,8-2,4)$ & 2,1 & $2,8(1,3-6,1)^{\#}$ \\
\hline $6,9-7,8$ & 4,9 & $1,8(1,0-2,9)$ & 2,8 & $3,8(1,9-7,3)^{\#}$ \\
\hline $7,9-8,8$ & 5,0 & $1,8(0,9-3,3)$ & 3,3 & $4,4(2,0-9,4)^{\#}$ \\
\hline $8,9-10,3$ & 3,9 & $1,4(0,6-3,1)$ & 6,3 & $8,3(4,2-15,9)^{\#}$ \\
\hline$\geq 10,4$ & 10,9 & $3,9(1,8-7,8)^{\#}$ & 5,5 & $7,3(2,5-19,8)^{\#}$ \\
\hline Durchschnittsbevölkerung & 2,8 & 1,0 & 0,75 & 1,0 \\
\hline
\end{tabular}

ist das Risiko für Morbidität und Mortalität deutlich erhöht (Tab. 1, Abb. 1). Die perikonzeptionelle Stoffwechselkontrolle und eine Nephropathie waren in einer populationsbasierten Kohortenstudie die wichtigsten unabhängigen Prädiktoren für das Auftreten von kindlichen Anomalien, die insgesamt 3- bis 6-fach häufiger waren als bei nicht-diabetischen Schwangeren [8]. Dabei stieg das Fehlbildungsrisiko linear an (nahezu $30 \%$ per $1 \% \mathrm{HbA}_{1 \mathrm{c}}$-Anstieg über $6,3 \%$ ). Das Hypoglykämierisiko ist insbesondere im 1. Trimenon sehr hoch und muss individuell berücksichtigt werden, sinkt dann aber mit zunehmender Insulinresistenz deutlich. In einem systematischen Review wurde pro $1 \% \mathrm{HbA}_{1 \mathrm{c}}$-Absenkung eine Reduktion des relativen Risikos für kongenitale Fehlbildungen um 0,39-0,59 bei Frauen mit T1DM oder T2DM ermittelt [9]. Um eine normoglykämische Stoffwechsellage zu erreichen, müssen die Frauen in funktioneller Insulintherapie geschult oder in der Pumpentherapie erfahren sein. Prinzipiell sind die beiden Therapieformen in ihrer Effektivität vergleichbar. Dies wird in einem Cochrane Review bei allgemein schlechter Studienqualität bestätigt [10]. Aufgrund des Fortschritts in der Pumpentechnologie wird die Durchführung von neuen, qualitativ hochwertigen Studien empfohlen. Eine multizentrische Studie mit fast 400 Schwangerschaften mit Frauen mit Typ 1 Diabetes zeigte unter einer Pumpentherapie Im Vergleich $\mathrm{zu}$ einer Basis-Bolus-Therapie niedrigere $\mathrm{HbA}_{1 \mathrm{c}}$-Werte bei vergleichbarem Hypoglykämierisiko [11]. Eine rezente Analyse der CONCEPTT Studie zeigte allerdings ein schlechteres Outcome von schwangeren Frauen mit Insulinpumpentherapie im Vergleich zu Basis-Bolus Therapie [12]. Unter einer intensivierten Basis Bolus Therapie wurden bessere glykämische Parameter, sowie ein geringeres Risiko für Schwangerschaftshypertonie, neonatale Hypoglykämie und Aufnahmen auf die neonatale Intensivstation festgestellt.

Komplexere Therapiekonzepte sollten bereits frühzeitig präkonzeptionell bei Kinderwunsch geschult und erlernt werden, um eine Gravidität bei stabiler normoglykämischer Stoffwechsellage zu ermöglichen. Der Einsatz der Pumpentherapie in Kombination mit Continuous Glucose Monitoring System (CGMS) Messungen kann die Einstellung in der Schwangerschaft erleichtern. In der erst kürzlich publizierten CONCEPTT Studie konnte bei der Verwendung von CGMS in der Schwangerschaft oder der Zeit der Schwangerschaftsplanung bei Frauen mit T1DM gezeigt werden, dass ein besseres neonatales Outcome im Vergleich zur kapillären Blutzuckerselbstmessung mit signifikant weniger LGA Geburten, Hypoglykämien und Aufenthalten auf der neonatologischen Intensivstation und kürzerem Spitalsaufenthalt verbunden ist [13]. Ebenso konnte eine signifikant geringere Zeit in der Hyperglykämie und dabei mehr Zeit im Zielbereich, bei nur minimalen Änderungen des $\mathrm{HbA}_{\mathrm{lc}}$ und vergleichbaren Hypoglykämieraten beobachtet werden. Die sichere und verlässliche Anwendung eines Flash Glucose Systems im Vergleich zu Blutzuckerselbstmessung wurde in der Schwangerschaft bei T1DM, T2DM und Gestationsdiabetes beobachtet [14].

Bei bestehendem Kinderwunsch ist eine Abklärung diabetischer Spätkomplikationen dringend erforderlich (Tab. 2). Eine Kontrolle des Augenhintergrundes beim Spezialisten, Kontrolle der Nierenfunktion und falls erforderlich weitere Abklärung durch einen spezialisierten Facharzt, die Einstellung des Blutdrucks mit in der Schwangerschaft geeigneten Medikamenten (potentiell teratogene Eigenschaften von Medikamenten wie ACE Hemmer, AT1-Rezeptor-Blocker, Statine, usw. beachten), eine Abklärung koronarer Herzkrankheit, Dyslipidämie, ein Ausschluss einer Schilddrüsenfunktionsstörung, sowie eine Gewichtsreduktion bei Adipositas sollten bei Kinderwunsch bestenfalls vor dem Absetzen von Verhütungsmitteln erfolgen $[3,15]$. Im ersten Trimenon sollte ein TSH Wert unter 2,5 $\mu \mathrm{U} / \mathrm{L}$ angestrebt werden, in jedem Fall aber ist bei schwangeren Frauen mit positiven TPO Antikörpern oder TSH Werten über $10 \mu \mathrm{U} / \mathrm{L}$ ein sofortiger Therapiebeginn mit Schilddrüsen-Hormonen empfohlen [16].

\section{Gravidität}

Während der Schwangerschaft soll versucht werden, individualisiert die bestmögliche Stoffwechsellage unter Berücksichtigung der Hypoglykämie-Wahrnehmung und -häufigkeit, der individuellen Fähigkeiten so wie der Lebensumstände mit normoglykämen Blut- 
Abb. 1 Zusammenhang von perikonzeptionellem $\mathrm{HbA}_{1 \mathrm{c}}$ bei Frauen mit Diabetes und dem Risiko einer kongenitalen Fehlbildung (nach [6]). Das National Institute for Health and Clinical Excellence (UK) empfiehlt einen $\mathrm{HbA}_{1 \mathrm{c}}$ unter $6,1 \%$, die American Diabetes Association (USA) unter 7,0\% bei Frauen mit Diabetes, die einen Schwangerschaft planen. Bei einem $\mathrm{HbA}_{1 \mathrm{c}}$ über $10 \%$ regt das National Institute for Health and Clinical Excellence an, den Frauen mit Diabetes dringend von einer Schwangerschaft abzuraten. (Linie Risiko, Blaue Schattierung 95\% Konfidenzintervall)

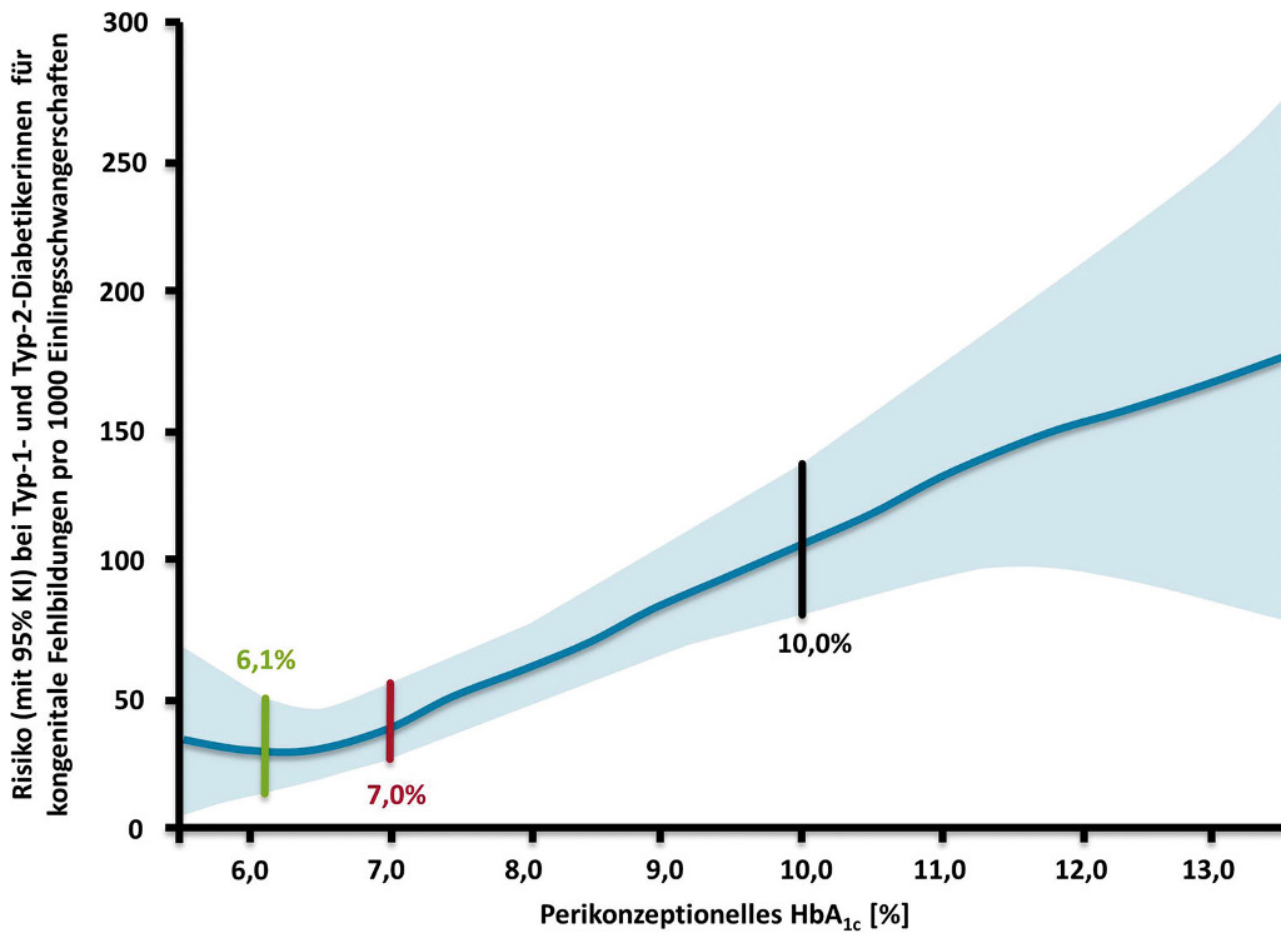

zuckerwerten (Tab. 3) zu erreichen. Lebensstilmaßnahmen mit regelmäßiger körperlicher Aktivität und Ernährungsumstellung sind auch in der Schwangerschaft zu empfehlen, und eine diätologische Beratung soll den schwangeren Frauen mit Diabetes angeboten werden (siehe Kapitel Gestationsdiabetes). Generell ist bei Frauen mit präkonzeptionellem Diabetes mellitus eine Insulintherapie in der Schwangerschaft zu empfehlen [5]. Gerade zu Beginn der Schwangerschaft ist die Hypoglykämierate relativ groß und die Insulindosis vorsichtig anzupassen. Insbesondere bei Frauen mit Typ 1 Diabetes ist das Risiko für schwere Hypoglykämien in der Frühschwangerschaft 3- bis 5-fach höher als vor der Schwangerschaft [17]. Generell gilt, dass im Lauf der Gravidität (üblicherweise beginnend mit der 20. Schwangerschaftswoche) die Tagesdosis auf 50-100\%, bei adipösen Frauen mit Typ 2 Diabetes oft noch höher angehoben werden müssen, um die zunehmende Insulinresistenz zu kompensieren und die empfohlenen Blutzuckerzielwerte in der Schwangerschaft zu erreichen (Tab. 3). Frauen mit T2DM und Kinderwunsch sollten bereits präkonzeptionell auf eine Insulintherapie umgestellt werden. Im Falle einer ungeplanten Gravidität bei T2DM unter Einnahme oraler Antidiabetika (OAD) gibt es bisher keine Evidenz für ein erhöhtes Missbildungsrisiko durch OAD, jedoch ist $\mathrm{zu}$ bedenken, dass Metformin plazentagängig ist und es bezüglich Langzeitfolgen bei den Nachkommen noch wenige Erkenntnisse vorliegen [5, 18]. Bei sehr insulinresistenten und stark übergewichtigen Frauen mit T2DM kann auch eine zusätzliche Therapie mit Metformin überlegt werden, um den Stoffwechsel $\mathrm{zu}$ verbessern und die Insulinresistenz zu mildern [5, 19]. Bei langzeitiger Einnahme von Metformin und Schwangerschaft wird eine Vitamin B12 Kontrolle empfohlen [18]. Eine Umstellung von OAD auf Insulin und eine entsprechende Schulung der Patientinnen zur Selbstanpassung der Insulindosis und Aufklärung über mögliche Risiken, sowie die $\mathrm{zu}$ erwartenden Stoffwechselveränderungen in der Schwangerschaft sollten durch die betreuenden Ärztinnen und Ärzte bei Schwangerschaftsplanung/ Kinderwunsch erfolgen. Bevorzugt werden die kurzwirksamen Insulinanaloga Insulin Aspart und Insulin Lispro verabreicht und mittlerweile in der Regel gegenüber Normalinsulin präferiert eingesetzt. Studien zeigen die sichere Anwendbarkeit von Insulin Lispro und Insulin Aspart in der Schwangerschaft [20, 21]. Das ultraschnell-wirksame Insulin Aspart (Fiasp ${ }^{\circledR}$ ) ist in der Schwangerschaft ebenso zugelassen. Für Glulisin liegen in der Gravidität derzeit nur Vigilanzdaten vor [22], die keine besonderen Auffälligkeiten zeigen. Aufgrund der schlechten Datenlage wird eine Anwendung in der Schwangerschaft nicht empfohlen.

Einige langwirksame Insulinanaloga sind in der Schwangerschaft zugelassen (Insulin Glargin, Insulin Glargin U300, Insulin Detemir) und können sicher angewendet werden, jedoch zeigten bisherige Daten keine eindeutigen Vorteile von Insulin Glargin oder Insulin Detemir gegenüber einer Therapie mit NPHInsulinen bei T1DM oder T2DM [23]. In einer Vergleichsstudie zwischen Detemir und NPH-Insulinen wurden vergleichbare $\mathrm{HbA}_{1 \mathrm{c}}$-Werte und ein ähnliches Hypoglyämierisiko beschrieben [23, 24]. Die Detemirgruppe konnte jedoch signifikant niedrigere Nüchternwerte in der 24. und 36. Schwangerschaftswoche 
Tab. 2 Übersicht: Erforderliche Maßnahmen vor und in der diabetischen Schwangerschaft (nach [15])

\begin{tabular}{|c|c|}
\hline \multirow[t]{2}{*}{ Insulintherapie } & Funktionelle Insulintherapie (Basal-Bolus-Prinzip) oder Insulinpumpe werden präferiert \\
\hline & Der Wechsel auf komplexere Dosierungsformen sollte möglichst vor Beendigung von Verhütungsmethoden erfolgen \\
\hline \multirow{2}{*}{$\begin{array}{l}\text { Hypoglykämie- } \\
\text { risiko }\end{array}$} & Kann limitierend für eine optimale Therapieeinstellung sein \\
\hline & Besonders in der Frühschwangerschaft bei T1DM ist das Risiko besonders hoch (3-5× erhöht) [17] \\
\hline Folsäure & Empfohlen: Beginn mit Folsäurepräparat drei Monate vor Beenden der Verhütung [15] \\
\hline \multirow[t]{3}{*}{ Augenkontrollen } & Kontrolle beim Spezialisten bei Kinderwunsch empfohlen (Fundus) \\
\hline & Bei Retinopathie ist falls erforderlich eine Therapieeinleitung durchzuführen. Der Kinderwunsch sollte bis zur Stabilisierung verzögert werden \\
\hline & Kontrolle: jedes Trimester, 3 Monate postpartum, danach je nach Erfordernis (zumindest $1 \times / J a h r)$ \\
\hline \multirow[t]{2}{*}{ Nierenfunktion } & Bei Niereninsuffizienz sollte ein spezialisierter Facharzt vor Absetzen der Verhütungsmethoden eine Risikoab- und -aufklärung durchführen \\
\hline & Regelmäßige Kontrollen in Schwangerschaft sind erforderlich \\
\hline \multirow[t]{3}{*}{ Blutdruck } & Zielwert: 130/80 mm Hg (Kontraindikation: ACE Hemmer + AT1-Rezeptor-Blocker) \\
\hline & Abklärung einer KHK, wenn vorhanden: Risikoab- und -aufklärung und gegebenenfalls Therapieeinleitung \\
\hline & Erhöhtes Präeklampsierisiko: Aspirin ${ }^{\circledR}(60-150 \mathrm{mg} / T a g)$ zur Prävention nach der 12. Schwangerschaftswoche bis zur Geburt empfohlen \\
\hline \multirow[t]{2}{*}{ Lipide } & Statine, Fibrate und Niacin kontraindiziert \\
\hline & $\begin{array}{l}\text { Gallesäurebindende Substanzen prinzipiell möglich, aber Nebenwirkungen (Gastrointestinaltrakt) beachten. Schwache Evidenz, in Kasuistiken } \\
\text { wurde außerdem ein erhöhtes Risiko von fetalen intrakraniellen Blutungen aufgrund eines Vitamin K Mangels beschrieben [30] }\end{array}$ \\
\hline \multirow{2}{*}{$\begin{array}{l}\text { Endokrine } \\
\text { Abklärung }\end{array}$} & Messung von TSH und TPO Antikörper vor Schwangerschaft \\
\hline & Bei Übergewicht: Gewichtsreduktion vor Schwangerschaft empfohlen (5-10\%) \\
\hline
\end{tabular}

Tab. 3 Blutglukose - Zielwerte in der Gravidität

\begin{tabular}{|l|l|}
\hline $\begin{array}{l}\text { Zeitpunkt der Blutzucker-Selbstmes- } \\
\text { sung }\end{array}$ & Blutzucker (mg/dl) \\
\hline Nüchtern und vor den Mahlzeiten & $60-90$ \\
\hline 1h nach Beginn der Mahlzeit & $<140$ \\
\hline 2h nach Beginn der Mahlzeit & $<120$ \\
\hline $\begin{array}{l}\text { Vor dem Schlafen gehen, } \\
\text { ca. } 22.00-23.00 \text { Uhr }\end{array}$ & $90-120$ \\
\hline Nachts in der Zeit von 2.00-4.00 Uhr & $>60$ \\
\hline
\end{tabular}

vorweisen [23]. Auch in der Analyse der perinatalen Komplikationen gab es zwischen den Insulin Glargin und Insulin Detemir vergleichbare Resultate [25]. Eine rezente Metaanalyse von Insulin Glargin Daten mit etwa 700 diabetischen schwangeren Frauen verglichen $\mathrm{zu}$ NPH zeigt vergleichbare maternale und kindliche Ergebnisse [26]. Insulin Degludec ist in der Schwangerschaft derzeit nicht zugelassen. Bisher gibt es zu Insulin Degludec in der Schwangerschaft nur Fallberichte, die keine negativen Outcomes beschrieben haben [27].

Während der Geburt sind Blutzuckerwerte zwischen $80-110 \mathrm{mg} / \mathrm{dl}$ anzustreben [28]. Nach Entbindung ist eine rasche Reduktion der Insulindosen um etwa 50\% und enge Blutzuckerkontrolle erforderlich, da die Insulinsensitivität rasch zunimmt [28].

Diabetische Folgeerkrankungen wie eine Retinopathie, Nephropathie oder autonome Neuropathie können fortschreiten, wobei die Veränderungen meist postpartal reversibel sind und im Langzeitverlauf somit üblicherweise durch die Gravidität selbst keine Progression eintritt. Frauen mit Nephropathie haben aber ein deutlich erhöhtes Risiko für die Entwicklung einer Präeklampsie und einer Frühgeburt sowie einer Wachstumsretardierung des Kindes. Im Falle von bereits vor der Schwangerschaft bestehenden Spätkom- plikationen muss eine Aufklärung der schwangeren Frau über ihr Risikoerfolgen. Während der Gravidität und postpartal sollte eine engmaschige, regelmäßige Beobachtung der Patientin durchgeführt werden (Tab. 2). Die Einnahme von Folsäure (mindestens $400 \mu \mathrm{g} / \mathrm{Tag}$ ) bereits bei Kinderwunsch bis einschließlich der 12. Schwangerschaftswoche ist obligat. Bei Adipositas oder T2DM werden bis zur 12. Schwangerschaftswoche sogar höhere Dosen $(5 \mathrm{mg})$ empfohlen [29].

Diabetes in der Schwangerschaft ist mit einem erhöhten Präeklampsierisiko verbunden. Daher sollte eine präventive Anwendung von niedrig dosiertem Aspirin (60-150 mg/Tag) nach der 12. Schwangerschaftswoche bis zur Geburt begonnen werden, um die Morbidität, Mortalität und auch Kosten zu senken [5].

Funding Open access funding provided by Medical University of Vienna.

Interessenkonflikt A. Kautzky-Willer hat von folgenden Unternehmen, die auch fördernde Mitglieder der ÖDG sind, Forschungsunterstützungen/Honorare erhalten: Eli Lilly, Novo Nordisk, Sanofi-Aventis. J. Harreiter hat von folgenden Unternehmen, die auch fördernde Mitglieder der ÖDG sind, Forschungsunterstützungen/Honorare erhalten: Astra Zeneca, Novo Nordisk, Takeda. Y. Winhofer-Stöckl hat von folgenden Unternehmen, die auch fördernde Mitglieder der ÖDG sind, Forschungsunterstützungen/Honorare erhalten: Sanofi Aventis, Eli Lilly, Astra Zeneca, Novartis. R. Weitgasser hat von folgenden Unternehmen, die teils auch fördernde Mitglieder der ÖDG sind, Forschungsunterstützungen und/oder Honorare erhalten: Abbott, Allergan, Astra Zeneca, Boehringer-Ingelheim, Dexcom, Eli Lilly, MSD, Novo Nordisk, Roche, Sanofi, Servier, Takeda. M. Lechleitner hat von folgenden Unternehmen, die auch fördernde Mitglieder der ÖDG sind, Honorare erhalten: Astra Zeneca, Bristol-Myers Squibb, Germania Pharmazeutika, GlaxoSmithKline Phar- 
ma, Eli Lilly, Medtronic, Merck Sharp\&Dome, Novartis, Novo Nordisk, Pfizer, Sanofi-Aventis, Takeda.

Open Access Dieser Artikel wird unter der Creative Commons Namensnennung 4.0 International Lizenz (http:// creativecommons.org/licenses/by/4.0/deed.de) veröffentlicht, welche die Nutzung, Vervielfältigung, Bearbeitung, Verbreitung und Wiedergabe in jeglichem Medium und Format erlaubt, sofern Sie den/die ursprünglichen Autor(en) und die Quelle ordnungsgemäß nennen, einen Link zur Creative Commons Lizenz beifügen und angeben, ob Änderungen vorgenommen wurden.

Hinweis des Verlags Der Verlag bleibt in Hinblick auf geografische Zuordnungen und Gebietsbezeichnungen in veröffentlichten Karten und Institutsadressen neutral.

\section{Literatur}

1. Kitzmiller JL, Block JM, Brown FM, et al. Managing preexisting diabetes for pregnancy: summary of evidence and consensus recommendations for care. Diabetes Care. 2008;31(5):1060-79. https://doi.org/10.2337/dc08-9020.

2. Feig DS, Palda VA. Type 2 diabetes in pregnancy: a growing concern. Lancet. 2002;359(9318):1690-2. https://doi.org/ $10.1016 / \mathrm{s} 0140-6736(02) 08599-9$.

3. Harreiter J, Kautzky-Willer A. Diabetes und Schwangerschaft. Wien Klin Wochenschr. 2016;11(1-4):23-42.https:// doi.org/10.1007/s11812-016-0078-9.

4. Handisurya A, Bancher-Todesca D, Schober E, et al. Risk factor profile and pregnancy outcome in women with type 1 and type 2 diabetes mellitus. J Womens Health (Larchmt). 2011;20(2):263-71.https://doi.org/10.1089/jwh.2010. 2033.

5. American Diabetes Association. 13. Management of diabetes in pregnancy: standards of medical care in diabetes2018. Diabetes Care. 2018;41(Suppl 1):S137-S43. https:// doi.org/10.2337/dc18-S013.

6. O'Brien SH, Koch T, Vesely SK, et al. Hormonal contraception and risk of thromboembolism in women with diabetes. Diabetes Care. 2017;40(2):233-8. https://doi.org/10.2337/ dc16-1534.

7. Jensen DM, Korsholm L, Ovesen P, et al. Peri-conceptional A1C and risk of serious adverse pregnancy outcome in 933 women with type 1 diabetes. Diabetes Care. 2009;32(6):1046-8. https://doi.org/10.2337/dc08-2061.

8. Bell R, Glinianaia SV, Tennant PW, et al. Peri-conception hyperglycaemia and nephropathy are associated with risk of congenital anomaly in women with pre-existing diabetes: a population-based cohort study. Diabetologia. 2012;55(4):936-47. https://doi.org/10.1007/s00125012-2455-y.

9. Inkster ME, Fahey TP, Donnan PT, et al. Poor glycated haemoglobin control and adverse pregnancy outcomes in type 1 and type 2 diabetes mellitus: systematic review of observational studies. BMC Pregnancy Childbirth. 2006;6:30. https://doi.org/10.1186/1471-2393-6-30.

10. Farrar D, Tuffnell DJ, West J, et al. Continuous subcutaneous insulin infusion versus multiple daily injections of insulin for pregnant women with diabetes. Cochrane Database Syst Rev. 2016; https://doi.org/10.1002/14651858. CD005542.pub3.

11. Kallas-Koeman MM, Kong JM, Klinke JA, et al. Insulin pump use in pregnancy is associated with lower HbAlc without increasing the rate of severe hypoglycaemia or diabeticketoacidosis in women with type 1 diabetes. Diabe- tologia.2014;57(4):681-9.https://doi.org/10.1007/s00125014-3163-6.

12. Feig DS, Corcoy R, Donovan LE, et al. Pumps or multiple daily injections in pregnancy involving type 1 diabetes: a prespecified analysis of the CONCEPTT randomized trial. Diabetes Care. 2018;41(12):2471-9. https://doi.org/ $10.2337 / \mathrm{dc} 18-1437$.

13. Feig DS, Donovan LE, Corcoy R, et al. Continuous glucose monitoring in pregnant women with type 1 diabetes (CONCEPTT): a multicentre international randomised controlled trial. Lancet. 2017;390(10110):2347-59. https:// doi.org/10.1016/S0140-6736(17)32400-5.

14. Scott EM, Bilous RW, Kautzky-Willer A. Accuracy, user acceptability, and safety evaluation for the freestyle libre flash glucose monitoring system when used by pregnant women with diabetes. Diabetes Technol Ther. 2018;20(3):180-8. https://doi.org/10.1089/dia.2017.0386.

15. Blumer I, Hadar E, Hadden DR, et al. Diabetes and pregnancy: an endocrine society clinical practice guideline. J Clin Endocrinol Metab. 2013;98(11):4227-49. https://doi.org/ $10.1210 /$ jc.2013-2465.

16. Alexander EK, Pearce EN, Brent GA, et al. 2017 guidelines of the American Thyroid Association for the diagnosis and management of thyroid disease during pregnancy and the postpartum. Thyroid. 2017;27(3):315-89. https://doi.org/ 10.1089/thy.2016.0457.

17. Ringholm L, Pedersen-Bjergaard U, Thorsteinsson B, et al. Hypoglycaemia during pregnancy in women with Type 1 diabetes. Diabet Med. 2012;29(5):558-66. https://doi.org/ 10.1111/j.1464-5491.2012.03604.x.

18. Lindsay RS, Loeken MR. Metformin use in pregnancy: promises and uncertainties. Diabetologia. 2017;60(9):1612-9. https://doi.org/10.1007/s00125-017-4351-y.

19. National Institute for Health and Care Excellence. Diabetes in pregnancy: management of diabetes and its complications from preconception to the postnatal period. NICE guideline (NG3). 2015.

20. Mathiesen ER, Kinsley B, Amiel SA, et al. Maternal glycemic control and hypoglycemia in type 1 diabetic pregnancy: a randomized trial of insulin aspart versus human insulin in 322 pregnant women. Diabetes Care. 2007;30(4):771-6. https://doi.org/10.2337/dc06-1887.

21. Di Cianni G, Volpe L, Ghio A, et al. Maternal metabolic control and perinatal outcome in women with gestational diabetes mellitus treated with lispro or aspart insulin: comparison with regular insulin. Diabetes Care. 2007;30(4):e11. https://doi.org/10.2337/dc06-2586.

22. Doder Z, Vanechanos D, Oster M, et al. Insulin Glulisine in pregnancy-experience from clinical trials and postmarketing surveillance. Eur Endocrinol. 2015;11(1):17-20. https://doi.org/10.17925/EE.2015.11.01.17.

23. Mathiesen ER, Hod M, Ivanisevic M, et al. Maternal efficacy and safety outcomes in a randomized, controlled trial comparing insulin detemir with NPH insulin in 310 pregnant women with type 1 diabetes. Diabetes Care. 2012;35(10):2012-7.https://doi.org/10.2337/dc11-2264.

24. Mathiesen ER, Damm P, Jovanovic L, et al. Basal insulin analogues in diabetic pregnancy: a literature review and baseline results of a randomised, controlled trial in type 1 diabetes. Diabetes Metab Res Rev. 2011;27(6):543-51. https://doi.org/10.1002/dmrr.1213.

25. Hod M, Mathiesen ER, Jovanovic L, et al. A randomized trial comparing perinatal outcomes using insulin detemir or neutral protamine Hagedorn in type 1 diabetes. J Matern Fetal Neonatal Med. 2014;27(1):7-13. https://doi.org/10. 3109/14767058.2013.799650. 
26. Lv S, Wang J, Xu Y. Safety of insulin analogs during pregnancy: a meta-analysis. Arch Gynecol Obstet. 2015;292(4):749-56. https://doi.org/10.1007/s00404-0153692-3.

27. Milluzzo A, Tumminia A, Scalisi NM, et al. Insulin degludec in the first trimester of pregnancy: report of two cases. J Diabetes Investig. 2017; https:// doi.org/10.1111/jdi.12721.

28. Feldman AZ, Brown FM. Management of type 1 diabetes in pregnancy. Curr Diab Rep. 2016;16(8):76. https://doi.org/ $10.1007 / \mathrm{s} 11892-016-0765-\mathrm{z}$.
29. Harreiter J, Schindler K, Bancher-Todesca D, et al. Management of pregnant women after Bariatric surgery. J Obes. 2018;4587064.https://doi.org/10.1155/2018/4587064.

30. Pharmakovigilanz- und Beratungszentrum für Embryonaltoxikologie, Institut für Klinische Pharmakologie und Toxikologie, Charité. Embryotox - Arzneimittelsicherheit in Schwangerschaft und Stillzeit. 2018. https://www. embryotox.de.Zugegriffen:7.Apr. 2018. 